\title{
Experiencia y reflexiones acerca de la protección de menores en el Reino Unido
}

\section{Experiences and reflections on child protection in the United Kingdom}

\author{
Marta LÓPEZ JIMÉNEZ \\ marta_lopez_jimenez@hotmail.es
}

Recibido: $24 / 09 / 2013$

Revisado: 27/11/2013

Aceptado: 26/05/2014

Disponible on line: $27 / 06 / 2014$

\section{Resumen}

Este artículo narra algunas de mis experiencias como trabajadora social en el Reino Unido. Abordaré la visión que en este contexto se tiene de esta profesión y de sus profesionales, como punto de partida para la reflexión acerca de aspectos concretos en la intervención social y el día a día de una trabajadora social, en un campo tan especifico como es el de la protección de menores. El artículo no pretende ser una generalización acerca de la intervención social en el Reino Unido, sino que pretende poner un ejemplo y abrir campos de reflexión acerca de las construcciones sociales y dinámicas que se generan sobre el Trabajo Social y su praxis en un campo tan particular como es el de la protección de menores.

Palabras clave: familia, marco, legislación, daño, proteger, Reino Unido.

\begin{abstract}
This article expresses some of my experiences as a social worker in the United Kingdom. In this paper, I will explore the way this field and its professionals are viewed in this specific context, before examining certain aspects of social intervention and the day to day job of a social worker, specifically in the field of child protection.

While this article does not try to be a generalization about social intervention in the United Kingdom, it attempts to give an example and open ideas of reflection around the social construction and dynamics around social work and its praxis in the particular field of child protection.
\end{abstract}

Keywords: family, framework, legislation, harm, safeguard, United Kingdom.

Referencia normalizada: López Jiménez, M. (2014): «Experiencia y reflexiones acerca de la protección de menores en el Reino Unido». Cuadernos de Trabajo Social, 27(1): 49-59.

Sumario: Introducción. 1. Imagen del Trabajo Social en los medios. 2. Protocolos de actuación. 3. Planes de menores en situación de necesidad. 4. Planes de protección de menores. 5. Medidas de protección inmediatas. 6. Revisión de casos «ejemplares» y sus implicaciones. 7. Lo que no se encuentra en el sistema, no existe. 8. Pasado, presente y futuro. 9. Conclusión. 10. Referencias bibliográficas.

\section{Introducción}

En el año 2011 me incorporé como trabajadora social en un Equipo de Apoyo al Menor y la Familia dentro del Ayuntamiento en un condado al sureste del Reino Unido, en donde trabajé durante dos años.

Fui contratada junto a más de 20 personas formando parte de un proceso de reclutamiento europeo de trabajadores sociales para trabajar en dicho condado. El resultado de éste, fue un gran número de trabajadores sociales ex- tranjeros, principalmente portugueses y rumanos, formando parte de los equipos de protección de menores en diferentes partes de esta comarca. El propio proceso de selección para este puesto en concreto, sirve de ejemplo para mostrar cómo encontrar trabajadores sociales dispuestos a trabajar en protección de menores en el Reino Unido es una tarea complicada. Según un estudio publicado por Community $\mathrm{Ca}$ re, uno de cada nueve puestos de trabajador social en 2009 estaba vacantes en el Reino Unido 
(Mickel, 2009). Trataré de reflexionar acerca de algunas de las causas de esto en el presente artículo.

Desarrollar tu ejercicio profesional como trabajadora social en el extranjero implica un gran reto tanto profesional como personal: nuevas legislaciones, protocolos, políticas, además de la evidente barrera idiomática y cultural a la que te enfrentas.

En mi caso concreto, la experiencia que adquirí en España durante mis años de formación y primeros años de carrera profesional en el ámbito de la intervención social con familias crearon en mí unas expectativas que no se correspondieron totalmente con la realidad de la intervención social en Inglaterra.

\section{Imagen del Trabajo Social en los medios}

La imagen que se proyecta de los trabajadores sociales en el Reino Unido, específicamente de aquellos que trabajan en el área de protección de menores, parece haber estado tradicionalmente teñida de negatividad, si lo miramos dentro del mundo del cine, la televisión o la prensa escrita.

La imagen que normalmente se tiene del Trabajo Social en el Reino Unido, como se destaca en los resultados de una encuesta realizada a trabajadores sociales en 2009 , es que los medios de comunicación tratan el Trabajo Social y a los trabajadores sociales de forma injusta, centrándose en todo aquello que se ve como fallos o injusticias «creadas» por ellos y solo transmitiendo noticas negativas acerca de estos profesionales (Jones, 2012).

Mis inquietudes acerca de mi futuro rol como trabajadora social en este ámbito profesional me llevaron a ver la película LadyBird, LadyBird (Loach, 1994). En esta película, basada en hechos reales, se cuenta la historia de una mujer que busca formar una familia y empezar de cero, tras haber tenido varios hijos e hijas tutelados por los Servicios Sociales. Sin embargo, se muestra como, una vez inmersa en la burocracia de los Servicios Sociales, esta tarea le resultará difícil.

Otro ejemplo que refleja esta imagen de los trabajadores sociales en los medios de comunicación puede observarse en la larga telenovela EastEnders (Smith y Holland, 1985) que se emite en el Reino Unido desde 1985. En un polémico capítulo, a una de las protagonistas se le retira la tutela de su hijo recién nacido sin pruebas aparentes que justifiquen que el menor se encuentra en riesgo inminente de desamparo de su madre.

Solo son dos ejemplos de la imagen de los trabajadores sociales que se ha venido proyectando en el cine y la televisión en el Reino Unido. Sin embargo, vemos como la prensa escrita ha tenido en particular un papel esencial a la hora de construir este retrato negativo de los trabajadores sociales de protección de menores.

Un estudio titulado, Trabajo Social en la prensa: un estudio transnacional, indica cómo mientras que en Estados Unidos la valoración de los trabajadores sociales es mayoritariamente positiva, en el Reino Unido se tiene una visión negativa de estos profesionales. En ambos contextos, se valora a los trabajadores sociales relacionados con la protección de menores más negativamente que esos mismos profesionales en otras áreas de intervención (Reid y Misener, 2001).

Jones (2012) indica que, específicamente en el periodo entre 2008 y 2010, fue cuando los trabajadores sociales del área de protección de menores aparecieron con más asiduidad en la prensa, debido a varios sucesos que habían ocurrido como: la muerte de dos menores, el secuestro de otro y la brutal agresión a otros dos. De algunos de los cuales hablaré más adelante en este artículo.

Un artículo publicado en esta época, Para proteger a los menores debemos proteger primero a los trabajadores sociales, plantea que es necesario revisar las expectativas que se tienen acerca de la labor de los trabajadores sociales. Este artículo indica que mientras es necesario aprender de las tragedias ocurridas y aceptar que el riesgo es inevitable, es también importante apreciar la resiliencia, el trabajo duro y las destrezas que los trabajadores sociales exhiben todos los días para asegurar que a muchos niños se les ayude de forma efectiva (Ferguson, 2008).

Mientras que observamos como el discurso mediático atiende a ciertas cuestiones, que no están directamente relacionadas con la justicia social, podemos observar también, tal y como indica Jones (2012), que ciertos periódicos se centraron en culpabilizar y buscar el escándalo, y este contexto abrió campos de debate y búsqueda de nuevas perspectivas sobre la protección de menores en el Reino Unido. 
Como veremos posteriormente, este contexto dio lugar a estudios específicos de expertos en el área de protección de menores, lo que implicó una serie de reformas en los protocolos de actuación a nivel estatal.

Aldridge (1990) presenta este constante debate entre Trabajo Social y medios de comunicación. Bien es cierto que existen ejemplos positivos en los medios de comunicación acerca de los trabajadores sociales, sin embargo, esta autora indica que suelen aparecen en la prensa lo$\mathrm{cal}$, mientras que a escala nacional tienen mayor eco las tragedias o el trabajo de organizaciones de voluntarios. La autora plantea igualmente que los intentos de las instituciones profesionales de reeducar a los medios de comunicación tendrán un éxito limitado y dichos esfuerzo deberán ser cuidadosamente enfocados.

En su libro Haciendo del Trabajo Social Noticia, esta misma autora plantea como a pesar de la percepción de la constante vulnerabilidad por parte de los trabajadores sociales, la realidad es que la mayoría de departamentos de servicios sociales no aparece en la prensa. En su opinión esto no es algo positivo, ya que de este modo sólo se tendrá en cuenta una serie de informaciones fragmentadas acerca de hechos reales o alegados en relación a escándalos o fallos en estos departamentos. Es por ello por lo que Aldridge (1994) plantea la necesidad de que los Servicios Sociales aparezcan de forma sistemática en las noticias, tanto cuando el sistema funciona como cuando se cometen ciertos errores.

Partiendo de esta reflexión, es interesante observar como recientemente la televisión pública inglesa ha emitido una serie de documentales titulados Protegiendo a nuestros niños (Burman, 2012), en los cuales se retrata la labor de los trabajadores sociales en equipos de protección de menores en Bristol. Estos documentales tienen el objetivo de mostrar el quehacer cotidiano de un trabajador social y advertir acerca de la complejidad de esta profesión, tantas veces juzgada por los medios de comunicación y por la población en general.

\section{Protocolos de actuación}

Para entender el contexto de esta intervención, es necesario referirse a los protocolos que rigen el trabajo con menores en los Servicios Sociales británicos. A continuación, trataré de resumir la vía de entrada y algunas de las diferentes situaciones que pueden producirse cuando un menor y su familia entran en contacto con los servicios de protección de menores.

Trabajando juntos para proteger a los menores. Guía de trabajo entre agencias para proteger y promocionar el bienestar de los niños (2010) establece que las diversas organizaciones e individuos deberán trabajar juntos con el fin de salvaguardar y promover el bienestar de niños y adolescentes, de acuerdo con la Ley del Menor de 1989 y la Ley del Menor de 2004.

Esta guía gubernamental se encuentra en constante revisión: en Marzo del 2013 se publicó una nueva versión actualizada. Comparaciones entre una y otra podrían servir de base para otro artículo, por lo que en el presente me basará en el documento de 2010, por tratarse del marco que guió mi práctica, durante los años que trabajé en este terreno.

Cualquier persona - ya sea vecino, familiar, miembro del público, en general- puede contactar con el departamento de menores de un Ayuntamiento si cree o tiene sospechas acerca de la situación en la que se encuentra un menor. Ciertamente los profesionales que trabajan con ese menor - profesores, médicos, etc. - tienen el deber de informar a los Servicios Sociales.

Siguiendo lo establecido por esta guía gubernamental, la rapidez con la que se lleve a cabo la evaluación de un menor dependerá de las necesidades particulares de dicho menor y de la naturaleza y el nivel de riesgo en que se halle. El departamento de protección de menores deberá tomar una decisión acerca del tipo de respuesta que se deba dar - así como documentarla en el sistema- dentro de las primeras 24 horas, tras ser notificados de un posible caso de desprotección. Esta decisión deberá tomarla el trabajador social contratándola con su supervisor.

Una vez se recibe ese primer contacto, el Equipo de Primera Valoración completará una primera evaluación de la situación en que se encuentra el menor, en un plazo máximo de 10 días. Esta valoración esencial determinará las necesidades actuales del menor, indicará si hay causas suficientes para creer que ese menor esté sufriendo o es probable que sufra un daño significativo y sugerirá unos servicios necesa- 
rios para salvaguardarle. La trabajadora social deberá ver al menor y, si es posible, se le tendrá que entrevistar a solas, para obtener su opinión y los detalles de lo ocurrido, con el fin de completar el informe preliminar. Dependiendo de la conclusión de esta valoración inicial, se pueden dar los siguientes escenarios:

- Fin de la intervención de Servicios Sociales. Tanto los profesionales como la familia consideran que el menor no requiere apoyo ni servicios específicos.

- Menores con necesidades no cubiertas.

- Menores en situaciones de riesgo o de riesgo probable.

- Menores con necesidad de protección inmediata.

Excepto en el primer caso, será necesario realizar una evaluación más completa con el objetivo de obtener información más detallada, y llevar a cabo un análisis de la situación del niño, que deberá completarse en un plazo de 35 días. Normalmente en este punto es cuando los casos se trasfieren al Equipo de Apoyo al Menor y a la Familia ${ }^{1}$.

Ambas, la evaluación inicial y la evaluación en profundidad, deben tener como punto de partida teórico un marco de evaluación, cuyos tres pilares esenciales son:

- Las necesidades que deben estar cubiertas para el buen desarrollo del menor, que incluyen: salud, educación, desarrollo de las emociones y comportamiento, identidad, familia y relaciones sociales, presentación social y habilidades de auto-cuidado.

- La capacidad de los padres para asegurar: cuidados básicos, seguridad, estimulación, guías y límites, estabilidad y cariño para el menor.

- Factores sociales y del entorno, tales como: historia y dinámicas familiares, familia lejana, vivienda, empleo, ingresos, integración social de la familia y acceso a recursos en la comunidad.
Estos tres pilares se representan en el siguiente triángulo de evaluación:

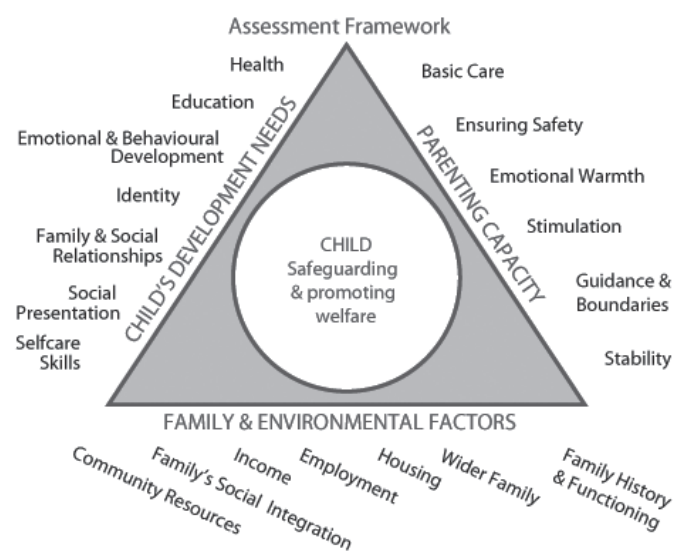

Working together to safeguard children (2013).

3. Planes de menores en situación de necesidad Según la sección 17 de la Ley del Menor 1989, bajo este concepto se encuentran:

- Menores para los cuales sería improbable alcanzar o mantener - o tener la oportunidad de alcanzar o mantener - un estándar razonable de salud o desarrollo sin la provisión de servicios por parte del Ayuntamiento.

- Menores cuya salud o desarrollo es probable que se vean significativamente perjudicados sin la provisión de servicios por parte del Ayuntamiento.

- Menores con una discapacidad substancial y permanente.

En estos casos, una vez concluida la valoración, se organizará una reunión donde la familia junto con el trabajador social y otros profesionales que intervienen con el menor, discutirán un plan de acción, con el fin de lograr unos objetivos que tratarán de ser específicos, medibles, alcanzables, realistas y dentro de un marco específico de tiempo, en beneficio del menor.

Para determinar la temporalidad con la que el trabajador social debe visitar al menor en su

\footnotetext{
${ }^{1}$ Como nota aclaratoria, se indica que tanto los nombres asignados a estos dos equipos como algunas de sus funciones están en constante revisión y cambio en los diferentes ayuntamientos. Es interesante observar que el Equipo de Apoyo al Menor y la Familia cambió recientemente de denominación, anteriormente se denominaba Equipo de Larga Duración. Ciertamente este cambio no es casual, sino que se trata de un intento consciente de enfatizar la labor del equipo más que el hecho de que, en muchos casos, la intervención con la familia puede dilatarse durante años.
} 
domicilio habrá que tenerse en cuenta la situación concreta y los riesgos específicos. Mientras que la guía anteriormente mencionada no establece un baremo temporal, es deber de cada región desarrollar sus propios protocolos a este respecto. En la región donde yo trabajaba debíamos visitar al menor al menos cada 4 semanas, con un máximo de 6 semanas de distancia entre visitas. Igualmente el plan debe revisarse en reuniones sucesivas con la familia y los profesionales, tal y como establece el Marco para la Evaluación de los Menores en situación de necesidad y sus Familias (2000) y es buena práctica revisar estos planes como máximo cada seis meses.

Sin embargo, la realidad muestra que en ciertos casos las familias no quieren verse involucradas con los Servicios Sociales y deciden no trabajar con ellos; por ejemplo, evitando las visitas o no acudiendo a las reuniones, etc. Teniendo en cuenta que en estos casos no hay sospechas de riesgo significativo para el menor, no se trata de un requerimiento legal y, por lo tanto, la familia puede optar por no querer apoyo profesional.

En ciertos casos, se trabaja con la familia desde un plan de menores en situación de necesidad ya que, aun existiendo un cierto nivel de riesgo para el menor, se considera que la situación no alcanza el parámetro necesario para ser considerado un caso de protección de menores. Sin embargo, en algunas de estas situaciones, el hecho de que la familia no quiera implicarse en el apoyo ofrecido puede ser indicativo de una falta de reconocimiento del problema para el menor $y$, a este punto, pueden aumentar las sospechas de un riesgo significativo. Ciertamente la evaluación del menor y su familia es un ejercicio dinámico, en el que es esencial la constante gestión y el análisis de riesgos.

El punto de inflexión entre una situación considerada de protección de menores y una de menores en situación de necesidad es la identificación del «daño significativo» para el menor. Tal como indica, Trabajando juntos para salvaguardar a los menores (2010), la Ley del Menor de 1989 introduce el concepto de daño significativo como el criterio que justifica la intervención obligatoria con una familia para la protección del menor. Éste confiere al Ayuntamiento la obligación de investigar acerca de la situación familiar para decidir si hay que llevar a cabo alguna acción para de proteger y promover el bienestar de un menor que está sufriendo o está en riesgo de sufrir un daño significativo. La sección 31 (9) de la Ley del Menor de 1989 define «daño», como maltrato o el impacto en la salud o el desarrollo del menor, incluyendo por ejemplo, el daño sufrido como consecuencia de percibir o escuchar el maltrato a otros.

Según dicha Guía, para entender e identificar este daño significativo en la vida de un menor, es importante considerar:

- El tipo de daño, relacionado con maltrato directo o con la falta de provisión de un cuidado adecuado al menor.

- El impacto que está teniendo en la salud y el desarrollo del menor.

- El desarrollo del menor dentro del contexto concreto de su familia y su entorno.

- Las necesidades específicas del menorenfermedad, discapacidad o problema de comunicación - lo cual puede afectar a su desarrollo $\mathrm{y}$ al cuidado recibido dentro de su familia.

- La capacidad de los padres para cubrir las necesidades del menor.

- El contexto familiar y social que le rodea.

\section{Planes de protección de menores}

Tal y como indica Trabajando juntos para salvaguardar a los menores (2010), cuando hay razones suficientes para sospechar que un menor está sufriendo o corre el riesgo de sufrir un daño significativo, los Servicios Sociales tendrán que convocar un «debate estratégico». Se trata de una reunión, presencial o telefónica, con otros departamentos tales como el de policía, departamentos de educación, salud, etc., con el fin de compartir información acerca de la situación del menor y determinar un plan que se ha de seguir. En esta fase la familia no está invitada, ya que se trata de una reunión multidisciplinar con el fin de acordar la mejor forma de proceder para proteger al menor. Durante esta reunión se discutirá si es necesario llevar a cabo una investigación conjunta con la policía.

Siguiendo esta investigación, y una vez llevadas a cabo las acciones acordadas en ese debate estratégico, se tomará una decisión acerca de la realidad de las sospechas iniciales. Si hay 
pruebas suficientes para creer que ese menor se halla en riesgo de sufrir daño significativo se procederá a un coloquio con Protección de Menores.

Una vez completada esta investigación y tomada la decisión de la necesidad de dicho coloquio, se organiza en los 15 días siguientes, tal y como se establece en Trabajando juntos para salvaguardar a los menores (2010). El objetivo del mismo es debatir, con la familia y todos los profesionales que trabajan con el menor, la situación actual y los riesgos, y acordar un plan de acción para salvaguardar al menor. Para este coloquio, el trabajador social deberá redactar un informe y lo habrá compartido con la familia antes de dicho coloquio. Éste lo presidirá un profesional independiente del departamento de menores, que se habrá reunido antes con la familia para escuchar su opinión sobre la situación que se va a discutir.

En este coloquio todos los profesionales implicados decidirán si el menor se halla en riesgo de daño significativo y la naturaleza de éste (negligencia, abuso físico, emocional o sexual). En caso de que así sea, el menor será inscrito en el Registro de Protección de Menores.

Al coloquio inicial, le seguirá otro, a los 3 meses, para revisar dicho plan. A partir de entonces se llevarán a cabo otras revisiones cada 6 meses. Entre unas y otras, la familia se reunirá también con los profesionales cada 6 semanas para revisar la marcha del plan. Siguiendo requerimientos legales, al menor le visitará regularmente el trabajador social. Como buena práctica, y según los procedimientos que yo debía seguir durante mi trabajo en ese campo, a los menores que se encuentran bajo un Plan de Protección el trabajador social debe visitarlos cada dos semanas, con visitas anunciadas o no anunciadas, pero en ellas el menor ha de hablar a solas y su habitación deberá poderla revisar el trabajador social.

\section{Medidas de protección inmediatas}

En caso de riesgo inminente para el menor, el Ayuntamiento tendrá que actuar lo antes posible para proteger al menor. En caso de que el menor se encuentre en riesgo inmediato y a falta de familiares o amigos que le puedan proveer con un cuidado adecuado, se planteará con la familia la opción de trasladar al menor temporalmente con una familia de acogi- da. En caso de que los padres con patria potestad estén en acuerdo con esta medida, el menor será trasladado bajo un acuerdo voluntaria, de acuerdo a la Sección 20 de la Ley del Menor de 1989. En este caso, la familia podrá requerir la vuelta a casa del menor en cualquier momento, ya que el Ayuntamiento no compartirá la patria potestad del menor si no media una orden judicial.

En caso de emergencia y falta de acuerdo por parte de la familia, el Ayuntamiento podrá solicitar una orden judicial de protección de emergencia, que será aplicada inicialmente durante 8 días, que conferirá al Ayuntamiento la capacidad de poner en marcha medidas que salvaguarden al menor durante ese tiempo concreto, incluido el traslado del menor a una familia de acogida. En los casos en los que no sea posible llevar a cabo este procedimiento, la policía podrá poner en marcha una orden de protección policial, que estará en vigor durante 72 horas.

En otras circunstancias, cuando los menores estén bajo un Plan de Protección y después de un cierto tiempo se considera que no han disminuido los riesgos o incluso que han aumentado, entonces el Ayuntamiento pedirá asesoramiento legal con el fin de salvaguardar al menor y solicitará, por la vía legal, la patria potestad del menor de forma permanente, que le conferirá al Ayuntamiento el poder de decisión sobre el futuro de ese menor.

\section{Revisión de los casos «ejemplares» y sus implicaciones}

Muchos de los procedimientos, documentos y guías que rigen la intervención social en materia de protección de menores han surgido tras la revisión de los casos «ejemplares». Son casos en los que la trágica muerte de un menor, a manos de sus familiares o cuidadores principales, ha cuestionado la eficacia de políticas y procedimientos y ha promovido cambios en el ámbito de la protección de menores.

En este sentido, dos de los casos de mayor transcendencia han sido la muerte de Victoria Climbié y de Peter Conelly (más conocido en la prensa como Baby P).

\section{Victoria Climbié}

Victoria Adjo Climbié era una niña que sufrió abusos y fue asesinada por sus cuidadores 
principales — su tía abuela y la pareja de ésta - en Londres en 2000, cuando tenía 8 años de edad.

Victoria fue quemada con cigarrillos, atada por periodos de más de 24 horas y herida con objetos, tales como cadenas y martillos. Antes de su muerte, tenían contacto con la niña los Servicios Sociales de cuatro distritos diferentes, los servicios de salud, una organización no gubernamental y la iglesia local a la que acudía la familia, y habían notado algunos signos de abuso en ella.

La indignación pública por su muerte dio lugar a una investigación que produjo grandes cambios en las políticas de protección de menores en el Reino Unido. Lord Laming condujo una investigación que dio lugar a la redacción de una guía llamada Cada niño importa (2003), la proclamación de la Ley del Menor de 2004, la creación del proyecto de ContactPoint - una base de datos gubernamental que contendría información de todos los niños en Inglaterra y Gales - y la creación de la Oficina del Comisionado de la infancia por el Comisario de la Infancia.

Cada niño importa (2003) fue una iniciativa gubernamental para Inglaterra y Gales, considerada una de las más importantes desarrollada en la última década en relación a los servicios de infancia, que incluye a todos los niños y jóvenes hasta 19 años, o hasta los 24 en el caso de jóvenes con discapacidad. Esta guía subraya la idea de que hay cinco objetivos esenciales que un niño debe alcanzar, sin que importe su origen o sus circunstancias. Estos son:

- Estar sano.

- Estar y sentirse seguro.

- Disfrutar y lograr objetivos.

- Hacer una contribución positiva.

- Conseguir bienestar económico.

En este documento se detalla un marco específico para cada una de estas áreas, cuyos objetivos requieren el trabajo conjunto de diferentes agencias - salud, educación, empleo, vivienda - para ser logrado.

Baby P.

Peter Conelly era un bebé de 17 meses que murió en Londres en Agosto del 2007, como consecuencia de más de cincuenta heridas que du- rante más de 8 meses, cuando residía con su madre, le infringió la pareja de su madre y otro adulto - que más tarde se descubrió que era el hermano de la pareja de la madre de Peter-. Durante este tiempo, al bebé lo vieron diferentes profesionales de los Servicios Sociales del distrito de Haringey y varios profesionales de los servicios sanitarios. La madre de Peter, su pareja y el hermano de éste fueron condenados por haber causado la muerte del bebe o haberlo permitido.

Esta muerte causó un gran impacto en la población general y en los partidos políticos por el nivel de las lesiones sufridas por el bebé y, entre otras razones, porque Peter vivía en el mismo distrito del norte de Londres donde 10 años antes tuvo lugar la muerte de Victoria Climbié. La revisión de este caso dio lugar a diversas medidas y esfuerzos conjuntos que se pusieron en marcha para que no volvieran a suceder hechos de esa envergadura.

Como consecuencia de ello, Lord Laming publicó un informe titulado La protección de menores en Inglaterra. Un informe de progreso (2009), donde indicaba que los servicios públicos no habían adoptado a tiempo las reformas introducidas y sugeridas por su anterior informe, tras la muerte de Victoria Climbié.

En 2011 se publicó un informe titulado, Revisión de la protección a la infancia en Inglaterra, una revisión independiente encargada por el gobierno a la profesora Munro. En él se examinaba el sistema de protección de menores y se presentaban algunas recomendaciones, con el fin de reformar el sistema vigente, pasando de uno caracterizado de superburocratizado a otro centralizase en el menor toda la intervención.

Entre sus conclusiones, la autora subrayaba la importancia de pasar de una cultura de la «culpa» a otra de aceptación del riesgo y la incertidumbre, permitiendo que los ayuntamientos desarrollaran sus propias propuestas acerca de la protección de menores. Indicaba que era necesario un cambio cultural sistemático y no la mera puesta en práctica de algunas de sus recomendaciones, aún siendo consciente de los retos surgidos debido a los recortes en la financiación y la constante reestructuración de servicios. Además, hacía hincapié en la idea de que dichos cambios ofrecían una oportunidad para cambios permanentes y a gran escala, pe- 
ro dependía de los ayuntamientos implementarlos de acuerdo con el espíritu de dicho informe (Munro, 2011).

En ambos casos, tanto en la muerte de Baby P como en la de Victoria Climbié, la falta de coordinación entre los profesionales y de una mirada crítica sobre ciertos hechos previos a la muerte de estos menores, fue fuertemente criticada y tuvo grandes implicaciones para las trabajadoras sociales responsables.

A la trabajadora social del distrito de Haringey que trabajó con Victoria la despidieron a raíz de la muerte de la menor. Tal y como indica un artículo publicado en Community Care aun cuando el informe de Lord Laming en 2003 indicó que la profesional no había sido correctamente supervisada a pesar de que su experiencia era escasa, no fue hasta 10 años después del fallecimiento de la menor cuando el General Social Care Council - cuerpo regulador de los trabajadores sociales en Inglaterra durante esta época- decidió que esta profesional podría volver a trabajar con menores, pero bajo condiciones como las de que debía volver a formarse o someterse a un reconocimiento médico (McGregor, 2010).

En el caso de Baby P, la trabajadora social del menor responsable del caso en el momento de su muerte y la jefa de ésta fueron despedidas del Ayuntamiento de Haringey. Ambas profesionales presentaron su caso ante un Tribunal de Trabajo para apelar esta decisión, sin embargo, el Tribunal dictó que su despido era justo, debido a su fallo en salvaguardar a dicho menor (BBC, 2013).

\section{Lo que no se encuentra en el sistema, no existe}

Los equipos de protección de menores —al igual que otros equipos dentro de los Servicios Sociales en el Reino Unido- cuentan con un sistema informático donde toda acción del trabajador social ha de estar documentada - detalles de visitas, actas de reuniones, valoraciones, contactos con otros profesionales, observaciones realizadas, decisiones tomadas por los jefes de equipo, etc., con las implicaciones consiguientes en lo que se refiere a la carga de trabajo diaria del profesional.

Y como se repite mucho en estos entornos: «lo que no se encuentra en el sistema, no existe», cuando se trata de proteger a personas vul- nerables es importante documentar que se han seguido los planes acordados, con los procedimientos y dentro de los plazos concretos. De esta manera en caso de que se lleve a cabo una auditoría sobre la situación específica de un menor, se pueda demostrar que tal y como está documentado ese determinado departamento está llevando a cabo todas las acciones necesarias, muchas de ellas acordadas en un entorno multidisciplinar.

Como indica Ferguson (2012), el actual sistema de recogida de datos, requiere que los trabajadores sociales empleen entre el 60 por ciento y el 80 por ciento de su jornada diaria en introducir datos en el sistema. Un dato sobrecogedor teniendo en cuenta que ese tiempo podría ser más efectivo si se utilizase para lo que ha llevado a esos trabajadores a dicha profesión: el trabajo con las personas.

Con el fin de gestionar y controlar que estos procedimientos se llevan a cabo, los jefes de equipo han de revisar folios de seguimiento en los que, de forma rutinaria, se recuerda a los profesionales las laboraeso que tienen que desempeñar: cuánto falta para completar una visita de protección de menores, qué tiempo falta para completar una valoración social, etc.

Es importante mencionar, que los equipos de protección de menores, al igual que otros servicios para menores, e instituciones educativas en el Reino Unido, se encuentran bajo la constante auditoría de la Oficina de Estándares en Educación y Servicios de Menores, más conocida como Ofsted. Dicho organismo es independiente, inspecciona y regula dichos servicios, emitiendo informes periódicos acerca de su calidad.

Según un artículo publicado por Community Care, en Junio del 2013, tras la reforma que llevó a cabo Ofsted - con el fin de incluir en el marco de las inspecciones de servicios de menores las recomendaciones sugeridas por el Informe Munro- el 25 por ciento de los 79 ayuntamientos inspeccionados en 2012 bajo este nuevo modelo, fueron calificados como «inadecuados», incluso aquellos con una reputación de buenas prácticas. Tal y como indica este artículo, muchos ayuntamientos opinaron que los inspectores de Ofsted se centraron en procesos - por ejemplo, en mantener unos registros escritos actualizados- más que en resultados (Cooper, 2013). 
Y todo ello implica grandes niveles de presión sobre los trabajadores sociales, de alguna forma los números pasan a cuestionar la intervención llevada a cabo con las familias. ¿Lleva un caso abierto demasiado tiempo para el departamento?, ¿lleva un menor demasiado tiempo bajo un plan de protección de menores? Algo parece que falla. De esta forma los números levantan alarmas entre los profesionales, lo que en algunos casos da la oportunidad para reflexionar, pero en otros, los menores se pierden del foco de atención para contemplar sobre todo números: de días, de casos, de protocolos...

\section{Pasado, presente y futuro}

Who cares? Un artículo publicado en 1994, tras la aparición de la película anteriormente citada, Ladybird Ladybird (Loach, 1994), analiza las opiniones que tienen de varios trabajadores sociales de ésta (German, 1994). Es interesante encontrar cómo aquellos trabajadores sociales retratados hace 20 años hablan de la necesidad de cambiar ciertas dinámicas dentro de los ayuntamientos, con el fin de promover una intervención social de calidad. Cambios que actualmente parece que no se han llevado a cabo completamente, y tienen grandes implicaciones en la intervención social con menores.

Como indica un estudio publicado en 2013, Reformar el trabajo social: mejorar el reclutamiento de trabajadores sociales, su formación y su retención, entre las causas que dificultan la retención de trabajadores sociales se encuentran: indicios de baja moral - con un promedio de bajas anual por enfermedad un 60 por ciento más alto que la media nacional-, corta vida laboral dentro de la profesión siendo 7.7 años para las mujeres y 8 años para los hombres - percepción de una gran carga de trabajo - una encuesta llevada a cabo por la Asociación Británica de Trabajadores Sociales identificó que un 70 por ciento de estos profesionales encontraban que su carga de trabajo era inmanejable - un gran número de sustituciones, oportunidades limitadas de promoción y excesivo trabajo administrativo (Holmes, Miscampbell y Robin 2013).

De tal forma que, una vez que se empieza a trabajar en este campo, es fácil observar cómo las presiones ejercidas por la gran cantidad de trabajo, la falta de recursos y de un equipo directivo estable y con capacidad de decisión, hace del trabajo social en equipos de protección de menores un campo muy exigente, debido a lo cual existe una gran rotación de los profesionales y una alta temporalidad. Esto hace que se emplee a muchos trabajadores sociales y jefes de equipo interinos. Tal y como indica Jones (2014), no es inusual que sea temporal entre el 40 por ciento y el 60 por ciento de la plantilla de uno de estos equipos. De este modo, menores y familias experimentan frecuentes cambios de trabajador social y las relaciones con otras agencias se ven deterioradas.

Y dentro de toda esta vorágine, nos podemos preguntar dónde queda el trabajo social con las comunidades. Se habla tanto del empoderamiento de las familias, pero ciertamente faltan recursos para trabajar desde las comunidades con aquellas familias que necesitan más apoyo, con una perspectiva no paternalista.

Durante mi labor como trabajadora social en esta área concreta, me he encontrado con profesionales de otros servicios, como centros para niños y familias, que ofrecen un gran apoyo y servicios, tales como: actividades para estimular a los menores, clases de apoyo para padres jóvenes, etc. - tristemente muchos de ellos se están viendo obligados a cerrar-. Tal y como indicaba Civil Society Media, 5 centros de Home Start — asociación con más de 330 sedes en Reino Unido, que ofrece apoyo a familias con hijos menores de 5 años - cerraban en 2011 mientras que otros 40 se encontraban en riesgo en el Reino Unido (Mair, 2011).

Al tiempo que se aprecia una falta de trabajo comunitario en este sistema de protección, podemos ver como ciertas intervenciones con familias concretas se «reabren» incesantemente en los Equipos de Protección de Menores. Ello podría sugerir que no se trabaja para llegar a la raíz del problema, con el fin de promover cambios estructurales, sino que más bien se promueve una intervención social en momento de crisis. $Y$ en este contexto sería necesario preguntarnos si este sistema tiene un espacio real para la prevención. Trabajando juntos para proteger a los menores (2010), aborda el marco de evaluación común, Common Assessment Framework, una herramienta que posibilita una evaluación temprana de menores y adolescente en situación de necesidad de apoyo y servicios. Se trata de un instrumento de 
evaluación cuyas implicaciones, efectividad y beneficios para los menores, por su extensión, podría ser tema para otro artículo.

Pero en un contexto como este, en el que el futuro parece estar cargado de desesperanza, encontramos también un escenario ideal para la reflexión y el surgimiento de nuevos modelos dentro de la intervención con menores. Un buen ejemplo lo encontramos en Re-imaginar la protección de menores: hacia el trabajo social humano con familias (Featherstone, Morris y White, 2014), una reciente publicación en la que se cuestiona la cultura de protección de menores que parece haber quedado atrapada en un autoritarismo contra las familias desfavorecidas. Según indica una de sus autoras, en un artículo publicado por Community Care:

El actual sistema no está sirviendo ni a los menores, ni a sus familias ni a los trabajadores sociales (...) Se ha convertido en un sistema muy reacio a los riesgos y demasiado obsesionado en sacar a menores de sus familias (Weir, 2014).

Esta autora sugiere la necesidad de crear equipos con un modelo centrado en las familias, desde los barrios, como oposición a un modelo individual de trabajador social.

\section{Conclusión}

En este artículo he querido reflexionar, tomando mi experiencia como punto de partida, acerca de la protección a la infancia en el Reino Unido. Nos encontramos en un contexto donde la culpabilización y el miedo parecen ser una constante, tanto como pauta de intervención con ciertas familias como en el trato hacia los propios trabajadores sociales.

En el Reino Unido encontramos una gran demanda de estos profesionales en el campo de la protección de menores. Algunas de las razo- nes que dificultan la retención de trabajadores sociales en este contexto son la excesiva carga de trabajo, la baja moral de los profesionales y el excesivo trabajo administrativo.

Hemos visto algunos de los procedimientos y protocolos que guían la intervención con menores, en los cuales el menor debe mantenerse como su foco principal, teniendo en cuenta sus necesidades en diferentes esferas- salud, educación, desarrollo emocional y comportamental, identidad, relaciones sociales y familiares, etc.- y entendiendo la valoración social del menor y su familia como un proceso, no como un ejercicio aislado. Un proceso en el que es una parte esencial el análisis de la información y de las observaciones realizadas, al mismo tiempo que un ejercicio arduo.

En este contexto, encontramos como la imagen de los trabajadores sociales promovida por los medios de comunicación ha estado cargada de sesgos negativos, haciendo especial hincapié en escándalos y dramas. Bien es cierto que en la actualidad, parece estar llevándose a cabo un esfuerzo consciente, por parte de algunos medios de comunicación, con el objetivo de mostrar la realidad de una profesión tan compleja, que requiere unos profesionales apasionados por su trabajo, conscientes de los retos y trabas que el sistema les pone delante, pero que luchan por hacer que las voces de los menores sean escuchadas.

Por todo ello, actualmente el sistema de protección de menores en el Reino Unido presenta un marco excepcional para desarrollar nuevos espacios de reflexión. Los cuales nos pueden llevar a cuestionar el presente modelo- como refleja el libro Re-imaginar la protección de menores (Featherstone, Morris y White, 2014) - y a reflexionar acerca de sus implicaciones presentes y futuras para los menores, sus familias y la sociedad en general.

\section{Referencias bibliográficas}

Aldridge, M. (1990). Social Work and the News Media: A Hopeless Case? The Bristish Journal of Social Work, 20 (6), 611-625.

Aldridge, M. (1994). Making Social Work News. Londres: Routledge.

BBC, News London (12 de marzo de 2013). Baby P social workers lose court appeal against sacking. Recuperado de: http://www.bbc.com/news/uk-england-london-21755808

Burman, E. (dir.). (2012). Protecting our children. Reino Unido. Bristol City Council.

Children Act 1989. (1989). Londres. H.M.S.O.

Children Act 2004. (2004). Londres. H.M.S.O. 
Cooper, J. (19 de junio de 2013). Quarter of councils 'inadequate' under new Ofsted inspection regime. Community Care. [On line] Recuperado de: http:/www.communitycare.co.uk/2013/ 06/19/quarter-of-councils-inadequate-under-new-ofsted-inspection-regime/\#.U3uQGvl_s04

Department of Health. (2000). Framework for the assessment of children in need and their families. The Stationery Office. Recuperado de: http://webarchive.nationalarchives.gov.uk/

Department for Education. (2003). Every child matters. The Stationery Office. Recuperado de: https://www.education.gov.uk

Department for Children, Schools and Families. . (2010). Working together to safeguard children. A guide to inter-agency working to safeguard and promote the welfare of children. The Stationery Office. Recuperado de: http://www.education.gov.uk/a

Department for Education. Working together to safeguard children. (2013). A guide to interagency working to safeguard and promote the welfare of children. The Stationery Office. Recuperado de: http://www.education.gov.uk/a

Featherstone, B., Morris K. y White, S. (2014). Re-imagining child protection Towards humane social work with families. Londres: Policy Press.

Ferguson, G. (13 de noviembre de 2008), To protect children we must first protect social workers. The Guardian. [On line] Recuperado de: http://www.theguardian.com/commentisfree/2008/ nov/13/child-protection-social-care

Ferguson, I. (2012). The Protection of Children in England: A Progress Report. Cuadernos de Trabajo Social, 25, 19-31

German, L. (1994, noviembre). Who cares? Socialist Review. [On Line], 180. Recuperado de: http://pubs.socialistreviewindex.org.uk/sr180/contents.htm

Holmes, E., Miscampbell, G. y Robin, B. (2013). Reforming Social Work: improving social worker recruitment, training and retention. (Policy Exchange). Londres. Recuperado de: http://www.policyexchange.org.uk/publications

Jones, R. (2012). Child protection, social work and the media: doing as well as being done to. Research, Policy and Planning, 29(2), 83-94. Recuperado de: http://ssrg.org.uk/wp-content/uploads/2012/01/JONES.pdf

Jones, R. (25 de abril de 2014), How can inadequate child protection services be improved? The Guardian. [On line] Recuperado de: http://www.theguardian.com/social-care-network/2014/ apr/25/improving-inadequate-child-protection-services

Laming, W. (2009). The protection of children in England: a progress report. The Stationery Offi$c e$. Londres: Department for children, schools and families.

Loach, K. (dir.). (1994). Ladybird, Ladybird. Reino Unido: Parallax Pictures Production.

Mair, V. (1 de septiembre de 2011), Five Home-Start charities close, with a further 40 at risk. Civil Society. [On line] Recuperado de: http://www.civilsociety.co.uk/finance/news/content/ 10353/five_home-start_charities_close_with_a_further_40_at_risk

McGregor, K. (5 de marzo de 2010), Arthurworrey wins 10-year battle for registration. Community Care. [On line] Recuperado de: http://www.communitycare.co.uk/2010/03/05/arthurworrey-wins-10-year-battle-for-registration/\#.U3tm8fl_s04

Mickel, A. (15 de abril de 2009), One in nine social work posts are vacant in England. Community care. [On line] Recuperado de: http://www.communitycare.co.uk/2009/04/15/one-in-ninesocial-work-posts-are-vacant-in-england/\#.U2-nMfl_s05

Munro, E. (2011). Munro review of child protection: final report. A child-centred system. (Department for Education) .Londres. The Stationery Office.

Reid, W. y Misener, E. (2001). Social work in the press: a cross-national study. International Journal of Social Welfare, 10(3), 194-201.

Smith, J. y Holland, T. (dirs.). (1985). EastEnders. Reino Unido: BBC Elstreet studios.

Weir, P., (17 de abril de 2014). Re-imagining Child Protection: New book proposes radical overhaul of 'flawed' system. Community Care. [On line] Recuperado de http://www.communitycare.co.uk/2014/04/17/re-imagining-child-protection-new-book-proposes-radical-overhaul-flawed-system/\#.U4DBA_1_s04. 